\title{
Soft gluon contributions to Drell-Yan and Higgs production beyond NNLO
}

\section{Ravindran*i}

Harish-Chandra Research Institute, Chhatnag Road, Jhunsi, Allahabad, India,

E-mail: ravindra@mri.ernet.in

\section{J. Smith}

C.N. Yang Institute for Theoretical Physics, Stony Brook University, Stony Brook, NY 11794-3840 USA.

E-mail: smith@max2.physics.sunysb.edu

We present threshold enhanced perturbative QCD corrections to rapidity distributions of DY, Higgs, $Z$ and $W^{ \pm}$bosons at hadron colliders. We have obtained the Sudakov resummed cross sections at $\mathrm{N}^{3} \mathrm{LO}$ level using renormalisation group invariance and the mass factorisation theorem that these hard scattering cross sections satisfy. We demonstrate how these higher order threshold QCD corrections stabilise the theoretical predictions at the LHC under variations of both renormalisation and factorisation scales.

8th International Symposium on Radiative Corrections (RADCOR)

October 1-5 2007

Florence, Italy

\footnotetext{
${ }^{*}$ Speaker.

†'We would like to thank Prof. Catani for invitation and support to participate RADCOR'07, GGI, Florence,Italy
} 
Recent theoretical advances in the computations of radiative corrections in perturbative Quantum Chromodynamics (pQCD) have lead to very accurate predictions for several important observables for physics at the Tevatron collider in Fermilab as well as at the upcoming Large Hadron Collider (LHC) in CERN [1]. The pQCD corrections to next-to-next-to leading $\operatorname{order}(N N L O)$ are known for total and differential cross sections for vector boson $\left(W^{ \pm}, Z\right)$, Drell-Yan, Higgs production (see $[2,3,4,5,6,7,8,9,10,11,12,13,14]$ ) which reduce theoretical uncertainties resulting from unknown renormalisation and factorization scales. The three-loop results for beta functions, anomalous dimensions and form factors $[15,16,17,18,19,20,21]$ have lead to predictions for the resummation up to $\mathrm{N}^{3} \mathrm{LL}$ [22, 23, 24, 25] as well as for the fixed order partial-soft-plus-virtual $\mathrm{N}^{3} \mathrm{LO}$ corrections to DY and Higgs total cross sections. The fixed order partial-soft-plus-virtual $\mathrm{N}^{3} \mathrm{LO}$ corrections $[22,25]$ to DY and Higgs production show the reliability of the perturbation theory results and demonstrate stability against the variations of renormalization and mass factorization scales. In this article we present threshold enhanced perturbative QCD corrections to rapidity distributions of DY, Higgs, $Z$ and $W^{ \pm}$bosons at hadron colliders using the Sudakov resummed cross sections at $\mathrm{N}^{3} \mathrm{LO}$ level. This is achieved using renormalisation group invariance and the mass factorisation theorem that these hard scattering cross sections satisfy.

The differential cross section for producing a vector boson or DY pair or Higgs can be expressed as:

$$
\frac{d^{2} \sigma^{J}}{d q^{2} d y}=\sigma_{\mathrm{Born}}^{J}\left(x_{1}^{0}, x_{2}^{0}, q^{2}\right) W^{I}\left(x_{1}^{0}, x_{2}^{0}, q^{2}\right),
$$

where $q$ is the four-momentum of the vector boson or DY pair or Higgs. In our case $q^{2}=M_{J}^{2}$ where $J=l^{+} l^{-}, H, Z, W^{ \pm}$but for convenience we use $q^{2}$ in the rest of the paper. Our normalisation is that $W_{\text {Born }}^{I}\left(x_{1}^{0}, x_{2}^{0}, q^{2}\right)=\delta\left(1-x_{1}^{0}\right) \delta\left(1-x_{2}^{0}\right)$. The superscript $I$ represents light-quarks $(q)$, gluons $(g)$ and heavy quarks $(b)$ but we only need $I=q, g$ for the rest of the paper. The $x_{i}^{0}(i=1,2)$ are related to $q^{2}$, the scaling variable $\tau=q^{2} / S$, and the rapidity $y$ of $J$ :

$$
y=\frac{1}{2} \log \left(\frac{p_{2} \cdot q}{p_{1} \cdot q}\right)=\frac{1}{2} \log \left(\frac{x_{1}^{0}}{x_{2}^{0}}\right), \quad \tau=x_{1}^{0} x_{2}^{0} .
$$

Here $S=\left(p_{1}+p_{2}\right)^{2}$ is the square of the hadronic center of mass energy and $p_{i}$ are the momenta of incoming hadrons $P_{i}(i=1,2)$.

In the QCD improved parton model, the function $W^{I}\left(x_{1}^{0}, x_{2}^{0}, q^{2}\right)$ can be written in terms of parton distribution functions (PDFs) appropriately convoluted with perturbatively calculable partonic differential cross sections $\left(\Delta_{d, a b}^{I}\right)$ as follows

$$
\begin{aligned}
W^{I}\left(x_{1}^{0}, x_{2}^{0}, q^{2}\right)= & \sum_{a, b=q, \bar{q}, g} \int_{0}^{1} d x_{1} \int_{0}^{1} d x_{2} \mathscr{H}_{a b}^{I}\left(x_{1}, x_{2}, \mu_{F}^{2}\right) \\
& \times \int_{0}^{1} d z_{1} \int_{0}^{1} d z_{2} \delta\left(x_{1}^{0}-x_{1} z_{1}\right) \delta\left(x_{2}^{0}-x_{2} z_{2}\right) \Delta_{d, a b}^{I}\left(z_{1}, z_{2}, q^{2}, \mu_{F}^{2}, \mu_{R}^{2}\right),
\end{aligned}
$$

where the subscript $d$ denotes the particular differential distribution (here, with respect to $y, x_{F}$ etc). Here $\mu_{R}$ is the renormalisation scale and $\mu_{F}$ the factorisation scale. The function $\mathscr{H}_{a b}^{I}\left(x_{1}, x_{2}, \mu_{F}^{2}\right)$ is the product of PDFs $f_{a}\left(x_{1}, \mu_{F}^{2}\right)$ and $f_{b}\left(x_{2}, \mu_{F}^{2}\right)$ renormalised at the factorisation scale $\mu_{F}$. That is,

$$
\mathscr{H}_{a b}^{q}\left(x_{1}, x_{2}, \mu_{F}^{2}\right)=f_{a}^{P_{1}}\left(x_{1}, \mu_{F}^{2}\right) f_{b}^{P_{2}}\left(x_{2}, \mu_{F}^{2}\right)
$$


with $x_{i}(i=1,2)$ the momentum fractions of the partons in the incoming hadrons.

We can express the partonic cross sections in terms of soft and hard parts. The soft parts come from gluons that appear in real emission as well as in the virtual processes. The infra-red safe contributions from the soft gluons can be obtained by adding the soft parts of the differential cross sections with the ultraviolet renormalised virtual contributions and performing mass factorisation using appropriate counter terms. These combinations are called the "soft-plus-virtual" (sv) parts of the differential cross sections. Hence we write

$$
\Delta_{d, a b}^{I}\left(z_{1}, z_{2}, q^{2}, \mu_{F}^{2}, \mu_{R}^{2}\right)=\Delta_{I, a b}^{\mathrm{hard}}\left(z_{1}, z_{2}, q^{2}, \mu_{F}^{2}, \mu_{R}^{2}\right)+\delta_{a b} \Delta_{d, I}^{\mathrm{sv}}\left(z_{1}, z_{2}, q^{2}, \mu_{F}^{2}, \mu_{R}^{2}\right), \quad I=q .
$$

The hard parts of the differential cross sections $\Delta_{I, a b}^{\text {hard }}\left(z_{1}, z_{2}, q^{2}, \mu_{F}^{2}, \mu_{R}^{2}\right)$ can be obtained by the standard procedure(see [26, 27]). The sv parts of the differential cross sections are obtained using the method discussed in the [25] so that

$$
\Delta_{d, I}^{\mathrm{sv}}\left(z_{1}, z_{2}, q^{2}, \mu_{R}^{2}, \mu_{F}^{2}\right)=\left.\mathscr{C} \exp \left(\Psi_{d}^{I}\left(q^{2}, \mu_{R}^{2}, \mu_{F}^{2}, z_{1}, z_{2}, \varepsilon\right)\right)\right|_{\varepsilon=0}
$$

where the $\Psi_{d}^{I}\left(q^{2}, \mu_{R}^{2}, \mu_{F}^{2}, z_{1}, z_{2}, \varepsilon\right)$ are finite distributions computed in $4+\varepsilon$ dimensions and they take the form

$$
\begin{aligned}
\Psi_{d}^{I}\left(q^{2}, \mu_{R}^{2}, \mu_{F}^{2}, z_{1}, z_{2}, \varepsilon\right)= & \left(\ln \left(Z^{I}\left(\hat{a}_{s}, \mu_{R}^{2}, \mu^{2}, \varepsilon\right)\right)^{2}+\ln \left|\hat{F}^{I}\left(\hat{a}_{s}, Q^{2}, \mu^{2}, \varepsilon\right)\right|^{2}\right) \delta\left(1-z_{1}\right) \delta\left(1-z_{2}\right) \\
& +2 \Phi_{d}^{I}\left(\hat{a}_{s}, q^{2}, \mu^{2}, z_{1}, z_{2}, \varepsilon\right)-\mathscr{C} \ln \Gamma_{I I}\left(\hat{a}_{s}, \mu^{2}, \mu_{F}^{2}, z_{1}, \varepsilon\right) \delta\left(1-z_{2}\right) \\
& -\mathscr{C} \ln \Gamma_{I I}\left(\hat{a}_{s}, \mu^{2}, \mu_{F}^{2}, z_{2}, \varepsilon\right) \delta\left(1-z_{1}\right) .
\end{aligned}
$$

The symbol " $\mathscr{C}$ " means convolution. For example, $\mathscr{C}$ acting on the exponential of a function $f\left(z_{1}, z_{2}\right)$ means the following expansion:

$$
\begin{aligned}
\mathscr{C} e^{f\left(z_{1}, z_{2}\right)=} & \delta\left(1-z_{1}\right) \delta\left(1-z_{2}\right)+\frac{1}{1 !} f\left(z_{1}, z_{2}\right)+\frac{1}{2 !} f\left(z_{1}, z_{2}\right) \otimes f\left(z_{1}, z_{2}\right) \\
& +\frac{1}{3 !} f\left(z_{1}, z_{2}\right) \otimes f\left(z_{1}, z_{2}\right) \otimes f\left(z_{1}, z_{2}\right)+\cdots
\end{aligned}
$$

In the rest of the paper the function $f\left(z_{1}, z_{2}\right)$ is a distribution of the kind $\delta\left(1-z_{j}\right)$ or $\mathscr{D}_{i}\left(z_{j}\right)$, where

$$
\mathscr{D}_{i}\left(z_{j}\right)=\left[\frac{\ln ^{i}\left(1-z_{j}\right)}{\left(1-z_{j}\right)}\right]_{+} \quad i=0,1, \cdots, \quad \text { and } \quad j=1,2,
$$

and the symbol $\otimes$ means the "double" Mellin convolution with respect to the variables $z_{1}$ and $z_{2}$. We drop all the regular functions that result from these convolutions when defining the sv part of the cross sections. The factors $Z^{I}\left(\hat{a}_{s}, \mu_{R}^{2}, \mu^{2}, \varepsilon\right)$ are the overall operator renormalisation constants. For the vector current $Z^{q}\left(\hat{a}_{s}, \mu_{R}^{2}, \mu^{2}, \varepsilon\right)=1$, but the gluon operator [28] gets overall renormalisation. The $\hat{F}^{I}\left(\hat{a}_{s}, Q^{2}, \mu^{2}, \varepsilon\right)$ are the standard form factors coming from the purely virtual parts of the cross sections [29]. In the form factors, we have $Q^{2}=-M_{J}^{2}$. The partonic cross sections depend on two 
scaling variables $z_{1}$ and $z_{2}$. The functions $\Phi_{d}^{I}\left(\hat{a}_{s}, q^{2}, \mu^{2}, z_{1}, z_{2}, \varepsilon\right)$ are called the soft distribution functions. The collinear singularities that result from the massless partons are removed using the mass factorisation kernels $\Gamma\left(z_{j}, \mu_{F}^{2}, \varepsilon\right)$ in the $\overline{\mathrm{MS}}$ scheme (see eqn.(7)). The unrenormalised (bare) strong coupling constant $\hat{a}_{s}$ is defined as $\hat{a}_{s}=\hat{g}_{s}^{2} / 16 \pi^{2}$, where $\hat{g}_{s}$ is the strong coupling constant which is dimensionless in $n=4+\varepsilon$ space time dimensions. The scale $\mu$ comes from dimensional regularisation which makes the bare coupling constant $\hat{g}_{s}$ dimensionless in $n$ dimensions.
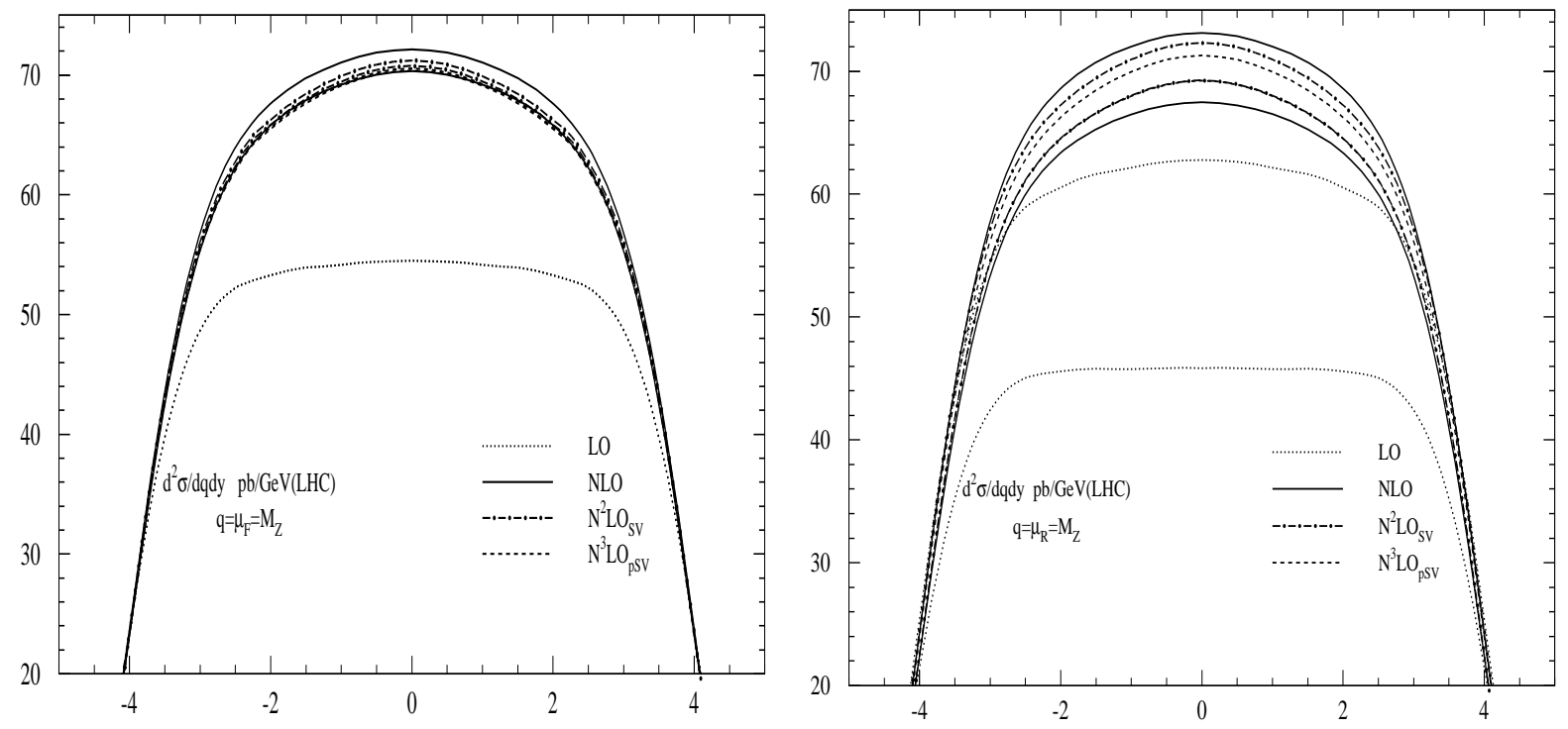

Figure 1: Rapidity distributions for $Z$ boson production at the LHC, and their $\mu=\mu_{R}$ (left panel) and $\mu=\mu_{F}$ (right panel) scale dependence (with $M_{Z}^{2} / 2<\mu^{2}<2 M_{Z}^{2}$ ). The abbreviation "pSV" means partial-soft-plusvirtual.

The fact that the $\Delta_{d, I}^{\mathrm{sv}}$ are finite in the limit $\varepsilon \rightarrow 0$ and that the form factors satisfy Sudakov differential equations leads to

$$
q^{2} \frac{d}{d q^{2}} \Phi_{d}^{I}\left(\hat{a}_{s}, q^{2}, \mu^{2}, z_{1}, z_{2}, \varepsilon\right)=\frac{1}{2}\left[\bar{K}_{d}^{I}\left(\hat{a}_{s}, \frac{\mu_{R}^{2}}{\mu^{2}}, z_{1}, z_{2}, \varepsilon\right)+\bar{G}_{d}^{I}\left(\hat{a}_{s}, \frac{q^{2}}{\mu_{R}^{2}}, \frac{\mu_{R}^{2}}{\mu^{2}}, z_{1}, z_{2}, \varepsilon\right)\right]
$$

where now the constants $\bar{K}_{d}^{I}$ contain all the singular terms in $\varepsilon$ and the $\bar{G}_{d}^{I}$ are finite functions of $\varepsilon$. The functions $\Phi_{d}^{I}\left(\hat{a}_{s}, q^{2}, \mu^{2}, z_{1}, z_{2}, \varepsilon\right)$ also satisfy the renormalisation group equations:

$$
\mu_{R}^{2} \frac{d}{d \mu_{R}^{2}} \Phi_{d}^{I}\left(\hat{a}_{s}, q^{2}, \mu^{2}, z_{1}, z_{2}, \varepsilon\right)=0
$$

The $\Phi_{d}^{I}\left(\hat{a}_{s}, q^{2}, \mu^{2}, z_{1}, z_{2}, \varepsilon\right)$ should contain the correct poles to cancel the poles coming from $\hat{F}^{I}, Z^{I}$ and $\Gamma_{I I}$ in order to make $\Delta_{d, I}^{\mathrm{sv}}$ finite. This requirement unambiguously determines all the poles of this distribution. The solution to the Sudakov differential equation for the soft distribution functions in eqn.(10) can be written as

$$
\Phi_{d}^{I}\left(\hat{a}_{s}, q^{2}, \mu^{2}, z_{1}, z_{2}, \varepsilon\right)=\sum_{i=1}^{\infty} \hat{a}_{s}^{i} S_{\varepsilon}^{i}\left(\frac{q^{2}\left(1-z_{1}\right)\left(1-z_{2}\right)}{\mu^{2}}\right)^{i \frac{\varepsilon}{2}}\left(\frac{(i \varepsilon)^{2}}{4\left(1-z_{1}\right)\left(1-z_{2}\right)}\right) \hat{\phi}_{d}^{I,(i)}(\varepsilon),
$$


where

$$
\hat{\phi}_{d}^{I,(i)}(\varepsilon)=\frac{1}{i \varepsilon}\left[\bar{K}_{d}^{I,(i)}(\varepsilon)+\bar{G}_{d}^{I,(i)}(\varepsilon)\right] .
$$

The constants $\bar{K}_{d}^{I,(i)}(\varepsilon)$ and $\bar{G}_{d}^{I,(i)}(\varepsilon)$ can be determined from the form factors, mass factorisation kernels, partonic cross sections and renormalisation constants.

Using this approach we have obtained the exact $\Delta_{d, I}^{\mathrm{sv},(\mathrm{i})}(I=q, g)$ up to $\mathrm{N}^{2} \mathrm{LO}(i=0,1,2)[11]$. The coefficient of the $\delta\left(1-z_{1}\right) \delta\left(1-z_{2}\right)$ part depends on the constants that are still unknown for $\mathrm{N}^{3} \mathrm{LO}$, so we can only obtain a partial result for $\Delta_{d, I}^{\mathrm{sv},(3)}$, i.e., a result without the $\delta\left(1-z_{1}\right) \delta\left(1-z_{2}\right)$ part can be computed from our formula given in eqn.(6). We can also obtain a result to $\mathrm{N}^{4} \mathrm{LO}$ order where we can predict partial sv contributions containing everything except the terms in $\mathscr{D}_{0}\left(z_{i}\right) \delta\left(1-z_{j}\right), \mathscr{D}_{0}\left(z_{i}\right) \mathscr{D}_{0}\left(z_{j}\right), \mathscr{D}_{1}\left(z_{i}\right) \delta\left(1-z_{j}\right)$ and $\delta\left(1-z_{1}\right) \delta\left(1-z_{2}\right)$ for the coefficient $\Delta_{d, I}^{\mathrm{sv},(4)}$. The convolutions of distributions of the form $\mathscr{D}_{l}\left(z_{j}\right) \otimes \mathscr{D}_{m}\left(z_{j}\right)$ for any arbitrary $l, m$ can be done using the general formulae given in [25] so we obtain $\Delta_{d, I}^{\mathrm{sv},(\mathrm{i})}$ for $i=1, \ldots, 4$.
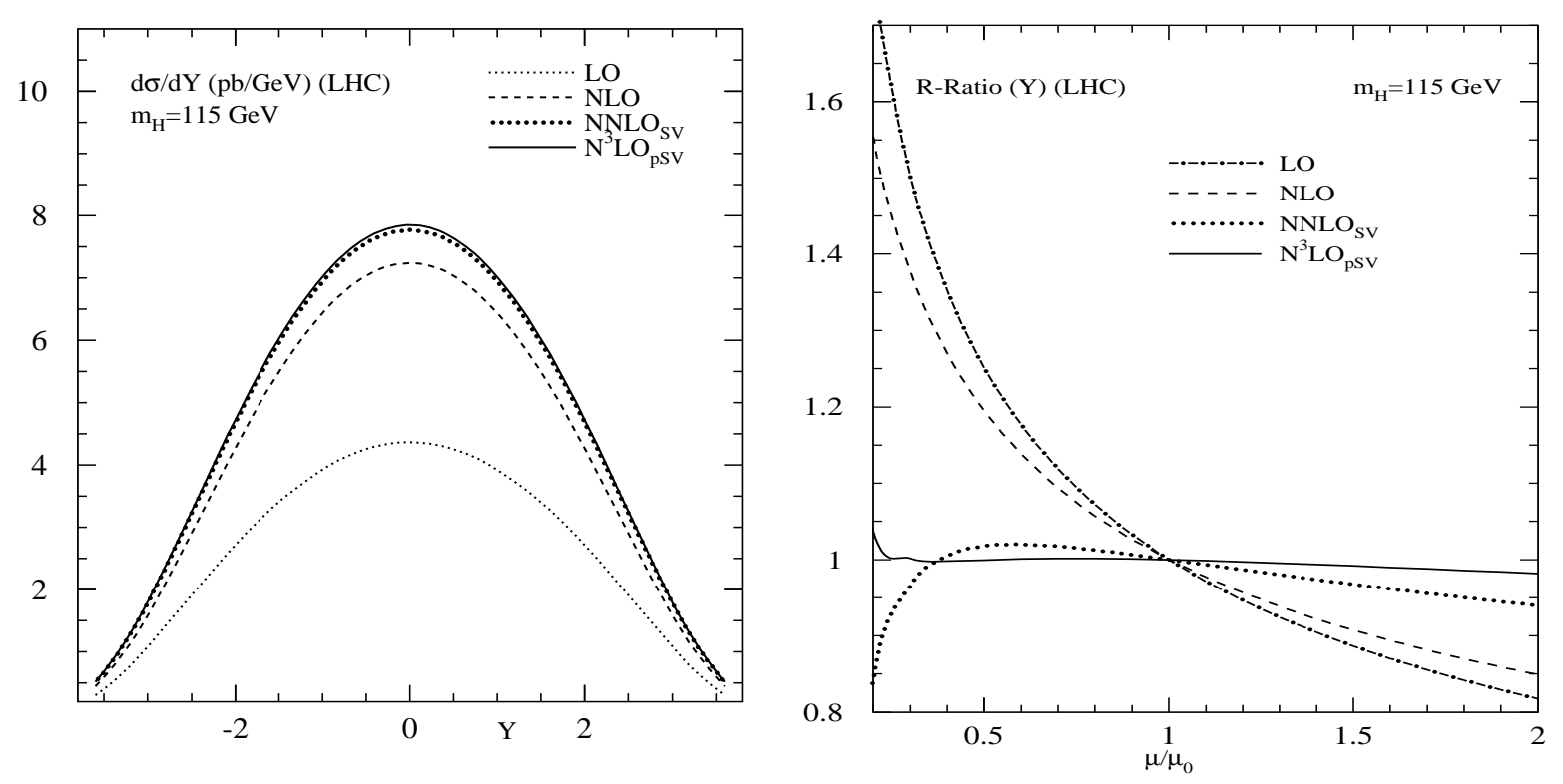

Figure 2: Rapidity distributions for Higgs boson production at the LHC with $\mu=\mu_{R}=\mu_{F}$ (left panel) and scale dependence (right panel)

We present here the numerical impact of our results on the rapidity distributions of $Z$ and Higgs production at LHC energies. Similar analysis can be done using our numerical code for DY pair, $W^{ \pm}$production for both $\mathrm{LHC}$ and Tevatron. We now give results for $Z$ production by choosing $q^{2}=M_{Z}^{2}$. The electro-weak constants relevant for our analysis can be found in [27, 9]. We present our results as differential cross sections in rapidity for fixed $q^{2}$ values.

We choose $\sqrt{S}=14 \mathrm{TeV}$ for the LHC, $Z$ boson mass $M_{Z}=91.19 \mathrm{GeV}$ and its width $2.50 \mathrm{GeV}$. We choose $\alpha_{s}^{\mathrm{LO}}\left(M_{Z}\right)=0.130, \alpha_{s}^{\mathrm{NLO}}\left(M_{Z}\right)=0.119, \alpha_{s}^{\mathrm{N}^{2} \mathrm{LO}}\left(M_{Z}\right)=0.115$ and $\alpha_{s}^{\mathrm{N}^{i} \mathrm{LO}}\left(M_{Z}\right)=0.113$ for $i>2$. The set MRST $2001 \mathrm{LO}$ is used for leading order, MRST2001 NLO for NLO and MRST 2002 NNLO for $\mathrm{N}^{i} \mathrm{LO}$ with $i>1$ [30, 31]. We use $\alpha=1 / 128$ for the electromagnetic fine structure constant, $\sin ^{2} \theta_{W}=0.2314$ for the weak mixing angle and $\cos ^{2} \theta_{C}=0.975$ for the Cabibbo angle. 
In fig. 1, we plot the rapidity distributions for the Z-boson at the LHC in LO (dotted lines), NLO (solid lines), $\mathrm{N}^{2} \mathrm{LO}$ (SV only, dot-dashed lines) and $\mathrm{N}^{3} \mathrm{LO}$ (pSV only, small-dashed lines). There are two panels in this plot and two curves in each panel since we show the scale variations by varying the mass factorization scale $\mu_{F}$ in the parton densities and the mass renormalization scale $\mu_{R}$ in the coefficient functions. Therefore we plot the curves at fixed $\mu_{F}^{2}=M_{Z}^{2}$ but with $\mu_{R}^{2}=M_{Z}^{2} / 2$ and $\mu_{R}^{2}=2 M_{Z}^{2}$ in the left panel and fixed $\mu_{R}^{2}=M_{Z}^{2}$ but with $\mu_{F}^{2}=M_{Z}^{2} / 2$ and $\mu_{F}^{2}=2 M_{Z}^{2}$ in the right panel. From the left panel we find that there is only a tiny dependence on the $\mu_{R}$ for fixed $\mu_{F}$ (here the LO result has no variation). In the right panel we find that $\mu_{F}$ dependence for fixed $\mu_{R}$ decreases as we go from LO to NLO, $\mathrm{N}^{2} \mathrm{LO}$ and $\mathrm{N}^{3} \mathrm{LO}$ respectively. The $\mathrm{N}^{3} \mathrm{LO}$ band lies within the $\mathrm{N}^{2} \mathrm{LO}$ band and both are within the bands for the NLO results. Notice that the lower curves for the $\mathrm{N}^{2} \mathrm{LO}$ and $\mathrm{N}^{3} \mathrm{LO}$ results fall on top of each other because the actual numbers are different but so close that one cannot see this from the plot. In fig. 2 we plot the rapidity distribution of Higgs boson at the LHC. The left panel is the distribution with $\mu_{R}=\mu_{F}=M_{H}$ and the right panel describes the sensitivity of the distribution with respect to the scale $\mu_{R}=\mu_{F}$. These results demonstrate that the perturbation series for the rapidity distribution converge very nicely at the LHC energy and the higher order results become less sensitive to renormalisation and factorisation scales.

To summarise, we have studied higher order sv corrections to rapidity distributions for DY, Higgs, $Z$ and $W^{ \pm}$boson production. We have used Sudakov resummation of soft gluons supplemented by renormalisation group invariance and the factorisation property of the observable. Using the available information on the form factors, the DGLAP kernels and lower order results we have obtained compact expressions for the resummation of soft gluons for the rapidity distributions of DY, Higgs, $Z, W^{ \pm}$boson. These allow us to compute sv rapidity distributions exactly at $\mathrm{N}^{2} \mathrm{LO}$ and partially at $\mathrm{N}^{3} \mathrm{LO}$. We have also presented the numerical impact of these results for $Z$ and Higgs bosons.

Acknowledgments: VR would like to thank Prof. Catani for the invitation and support to participate RADCOR'07, GGI at Florence and hospitality.

\section{References}

[1] M. Dittmar et al., "Parton distributions: Summary report for the HERA - LHC workshop," [arXiv:hep-ph/0511119].

[2] J. Kubar-Andre and F. E. Paige, Phys. Rev. D 19 (1979) 221.

[3] G. Altarelli, R. K. Ellis and G. Martinelli, Nucl. Phys. B 143 (1978) 521, [Erratum-ibid. B 146 (1978) 544].

[4] B. Humpert and W. L. van Neerven, Nucl. Phys. B 184 (1981) 225.

[5] T. Matsuura and W. L. van Neerven, Z. Phys. C 38 (1988) 623.

[6] T. Matsuura, S. C. van der Marck and W. L. van Neerven, Nucl. Phys. B 319, 570 (1989).

[7] R. Hamberg, W. L. van Neerven and T. Matsuura, Nucl. Phys. B 359 (1991) 343, [Erratum-ibid. B 644 (2002) 403].

[8] R. V. Harlander and W. B. Kilgore, Phys. Rev. Lett. 88 (2002) 201801 [arXiv:hep-ph/0201206]. 
[9] V. Ravindran, J. Smith and W. L. van Neerven, Nucl. Phys. B 665 (2003) 325 [arXiv:hep-ph/0302135].

[10] C. Anastasiou, L. J. Dixon, K. Melnikov and F. Petriello, Phys. Rev. Lett. 91 (2003) 182002 [arXiv:hep-ph/0306192].

[11] C. Anastasiou, L. J. Dixon, K. Melnikov and F. Petriello, Phys. Rev. D 69 (2004) 094008 [arXiv:hep-ph/0312266].

[12] K. Melnikov and F. Petriello, Phys. Rev. Lett. 96 (2006) 231803 [arXiv:hep-ph/0603182].

[13] K. Melnikov and F. Petriello, Phys. Rev. D 74 (2006) 114017 [arXiv:hep-ph/0609070].

[14] A. Cafarella, C.Coriano and M. Guzzi, [arXiv:hep-ph/0702244].

[15] S. Moch, J. A. M. Vermaseren and A. Vogt, Nucl. Phys. B 688 (2004) 101 [arXiv:hep-ph/0403192].

[16] A. Vogt, S. Moch and J. A. M. Vermaseren, Nucl. Phys. B 691 (2004) 129 [arXiv:hep-ph/0404111].

[17] S. Moch, J. A. M. Vermaseren and A. Vogt, JHEP 0508 (2005) 049 [arXiv:hep-ph/0507039].

[18] S. Moch, J. A. M. Vermaseren and A. Vogt, Phys. Lett. B 625 (2005) 245 [arXiv:hep-ph/0508055].

[19] J. A. M. Vermaseren, A. Vogt and S. Moch, Nucl. Phys. B 724, 3 (2005) [arXiv:hep-ph/0504242].

[20] S. Moch, J. A. M. Vermaseren and A. Vogt, Nucl. Phys. B 726 (2005) 317 [arXiv:hep-ph/0506288].

[21] J. Blumlein and J. A. M. Vermaseren, Phys. Lett. B 606 (2005) 130 [arXiv:hep-ph/0411111].

[22] S. Moch and A. Vogt, Phys. Lett. B 631 (2005) 48 [arXiv:hep-ph/0508265].

[23] E. Laenen and L. Magnea, Phys. Lett. B 632 (2006) 270 [arXiv:hep-ph/0508284].

[24] A. Idilbi, X. d. Ji, J. P. Ma and F. Yuan, Phys. Rev. D 73 (2006) 077501 [arXiv:hep-ph/0509294].

[25] V. Ravindran, Nucl. Phys. B 752 (2006) 173 [arXiv:hep-ph/0603041].

[26] P. J. Rijken and W. L. van Neerven, Phys. Rev. D 51 (1995) 44 [arXiv:hep-ph/9408366].

[27] P. Mathews, V. Ravindran, K. Sridhar and W. L. van Neerven, Nucl. Phys. B 713 (2005) 333 [arXiv:hep-ph/0411018].

[28] K. G. Chetyrkin, B. A. Kniehl and M. Steinhauser, Nucl. Phys. B 510 (1998) 61 [arXiv:hep-ph/9708255].

[29] V. Ravindran, J. Smith and W. L. van Neerven, Nucl. Phys. B 767 (2007) 100 [arXiv:hep-ph/0608308].

[30] A. D. Martin, R. G. Roberts, W. J. Stirling and R. S. Thorne, Phys. Lett. B 531 (2002) 216 [arXiv:hep-ph/0201127].

[31] A. D. Martin, R. G. Roberts, W. J. Stirling and R. S. Thorne, Eur. Phys. J. C 23 (2002) 73 [arXiv:hep-ph/0110215]. 